\title{
A Case of Cowden Syndrome with Bilateral Breast Cancer: Invasive Carcinoma of No Special Type and Pure Cribriform Carcinoma
}

\author{
Yoo Kyeong Seo ${ }^{1}$, Go Eun Yang ${ }^{1,}{ }^{*}$ and Taek Geun Ohk ${ }^{2}$ \\ ${ }^{1}$ Department of Radiology, Kangwon National University Hospital, Chuncheon, South Korea \\ ${ }^{2}$ Department of Emergency Medicine, School of Medicine, Kangwon National University Hospital, Chuncheon, South Korea \\ "Corresponding author: Department of Radiology, Kangwon National University Hospital, Chuncheon, South Korea. Email: yangkeoo@hanmail.net \\ Received 2019 April 03; Revised 2019 December 13; Accepted 2019 December 24.
}

\begin{abstract}
Cowden syndrome is an uncommon, multi-system disease that increases the risk of breast cancer. The most common breast malignancy is ductal carcinoma including ductal carcinoma in situ or infiltrating ductal carcinoma. Rare types of breast cancer exist, such as infiltrating tubular carcinoma and lobular carcinoma. There has been rarely a reported case of this type of breast cancer in Cowden syndrome. In this report, a 34-year-old woman diagnosed with Cowden syndrome underwent simple mastectomy for invasive carcinoma of no special type (NST) in the left breast, and simple mastectomy for cribriform carcinoma in the contralateral breast. Herein, we describe a case of Cowden syndrome with bilateral breast cancer, invasive carcinoma of NST in one breast and cribriform carcinoma in the contralateral breast.
\end{abstract}

Keywords: Breast, Cowden Syndrome, Cancer

\section{Introduction}

Cowden syndrome, or multiple hamartoma syndrome, is an autosomal dominant disorder involving hamartomatous overgrowth of tissues in various organs. Both benign and malignant conditions are common in Cowden syndrome. The risk of developing breast cancer is approximately $20 \%-50 \%$, which is three to four times higher than the general women population (1-5).

Breast cancer in Cowden syndrome is bilateral in nearly $25 \%$ of patients, and the pathology is ductal carcinoma in most cases. Tubular carcinoma and lobular carcinoma have rarely been reported $(2,6)$. Moreover, there have been no reports of pure cribriform carcinoma in cases of Cowden syndrome.

We recently encountered a woman with Cowden syndrome with invasive carcinoma of no special type (NST) in one breast and cribriform carcinoma in the contralateral breast.

\section{Case Presentation}

A34-year-old woman was diagnosed with multiple gastric polyps on upper endoscopy at the gastroenterologic medicine department and seborrheic hyperplasia on skin biopsy in our hospital. She had a history of multiple benign hemangiomas in the leg, abdomen, and palm 16 years prior. Therefore, she was referred to our breast clinic because of the suggestion of Cowden syndrome. At that time, she did not have any complaints, palpability or pain in her breasts. Phosphatase and tensin homolog (PTEN) DNA sequencing tests were confirmed as Cowden syndrome by showing a frameshift mutation.

She was referred to the department of surgery for evaluation of breast lesions. Extreme dense breast tissue with involuting fibroadenomas and benign calcifications were observed on mammography, which was American College of Radiology (ACR) Breast Imaging Reporting and Data System (BI-RADS) category 3 assessment (Figure 1 ).

On ultrasonography, a $1.8 \mathrm{~cm}$ irregular hypoechoic mass in the left breast with BI-RADS category 5 assessment (Figure 2A), and $2.7 \mathrm{~cm}$ large ovoid, lobulating mass in the right breast with BI-RADS category $4 \mathrm{~A}$ assessment (Figure 2B) were observed. Otherwise, there were multiple variable-sized, ovoid circumscribed nodules in both breasts. After diagnostic ultrasonography guided 14 G-core needle biopsy, the left breast lesion was diagnosed as invasive ductal carcinoma of no special type (NST) and the right breast lesion was infiltrative cribriform carcinoma. Low power microscopic findings in the right breast mass show pure infiltrating cribriform carcinoma (Figure 3 ). 

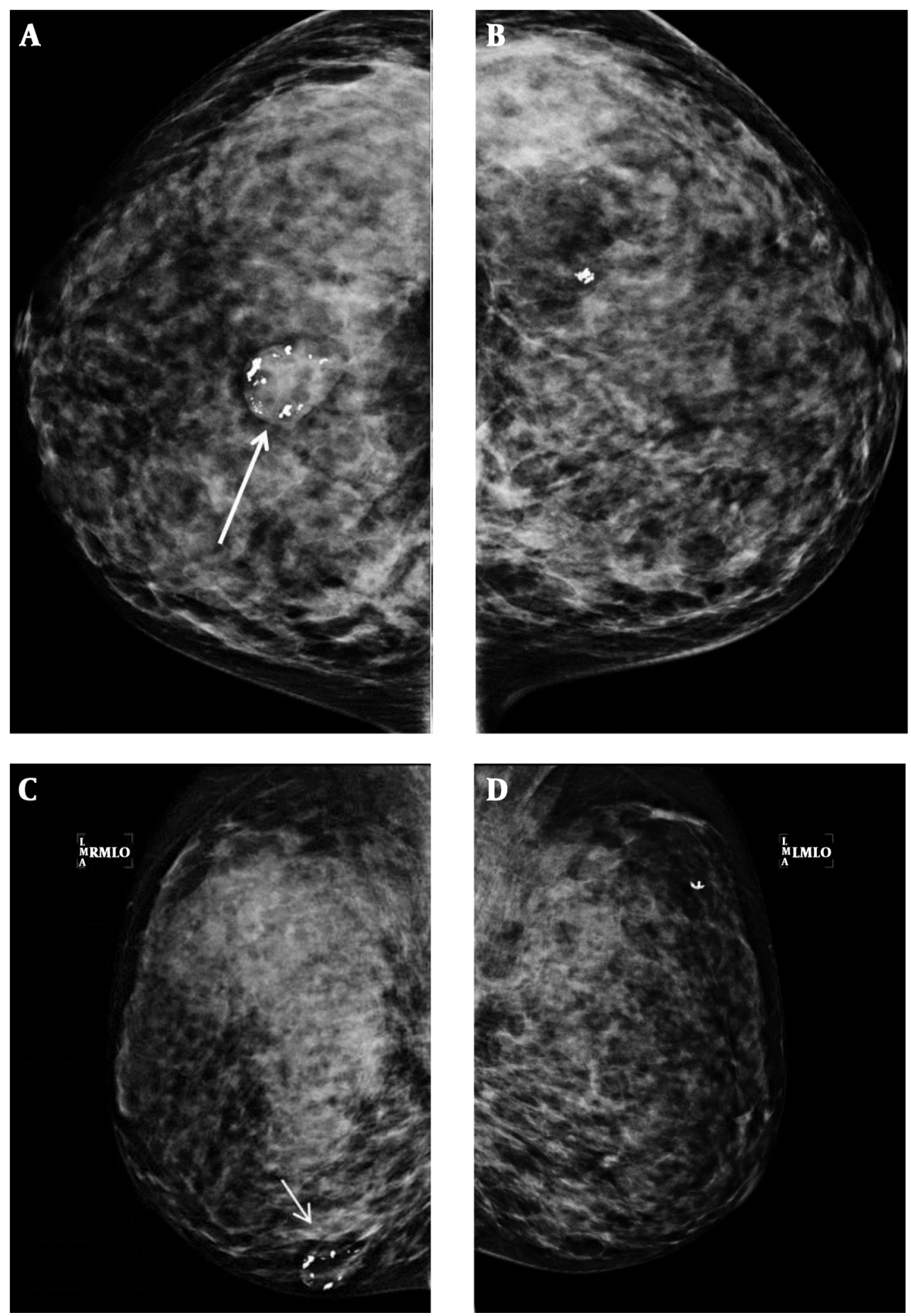

Figure 1. Bilateral routine views of mammography in a 34-year-old woman diagnosed with Cowden syndrome shows extreme dense breast tissue. Suggested involuting fibroadenoma (arrows) and benign calcifications are shown on the lower central portion of the right breast. A, Right craniocaudal view; B, Right mediolateral oblique view; C, Left craniocaudal view; D, Left mediolateral oblique view.

She underwent simple mastectomy of both breasts, and there were no metastases at the sentinel lymph nodes or distant organs. According to the American Joint Committee on Cancer, 8th edition, T1c in the right breast lesion and $\mathrm{T} 2$ in the left breast lesion without any lymph node metastasis, both tumors were low grade tumor (7). After surgery, she underwent chemotherapy and radiation therapy.

Regarding the thyroid gland, there were multiple, variable-sized, probably benign nodules including a follic- 

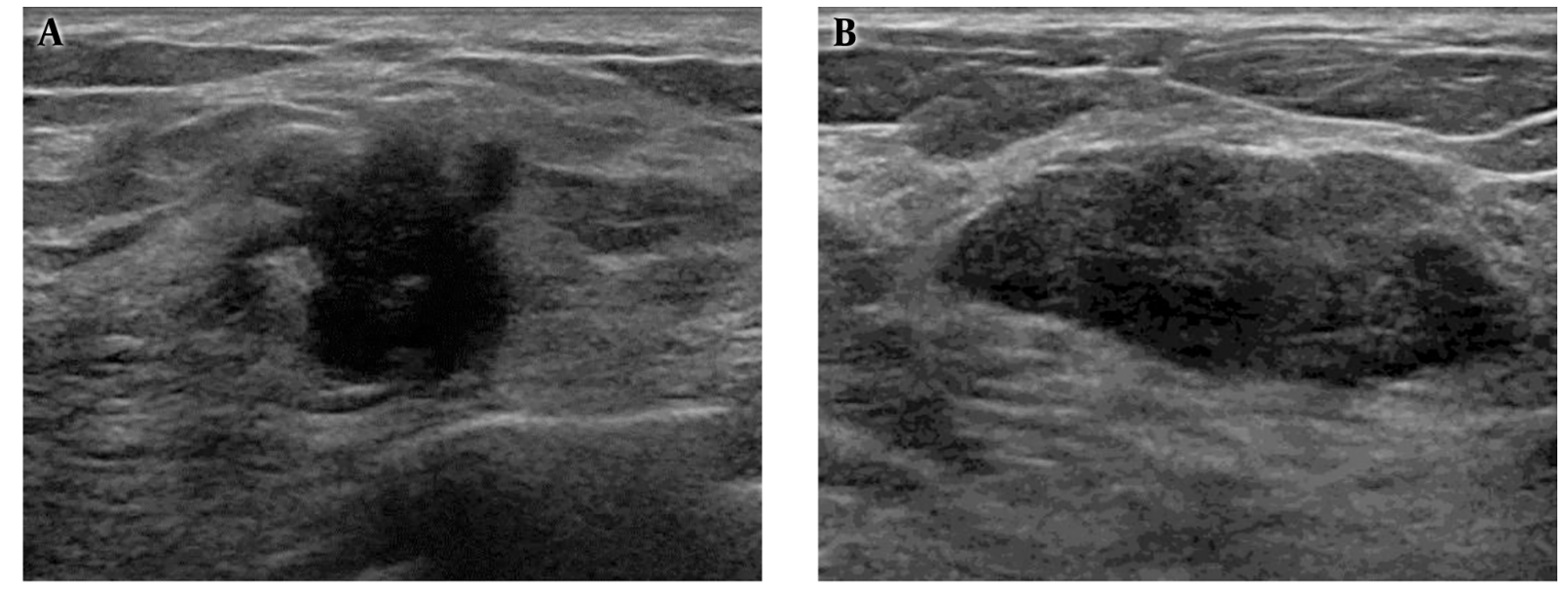

Figure 2. A, Ultrasonography shows a $1.8 \mathrm{~cm}$ sized irregular nonparallel hypoechoic mass in the left breast, highly suggestive of malignancy, and finally proven as invasive ductal carcinoma; B, A $2.7 \mathrm{~cm}$ sized ovoid, focal-lobulating contoured hypoechoic mass in the right breast diagnosed as cribriform carcinoma.

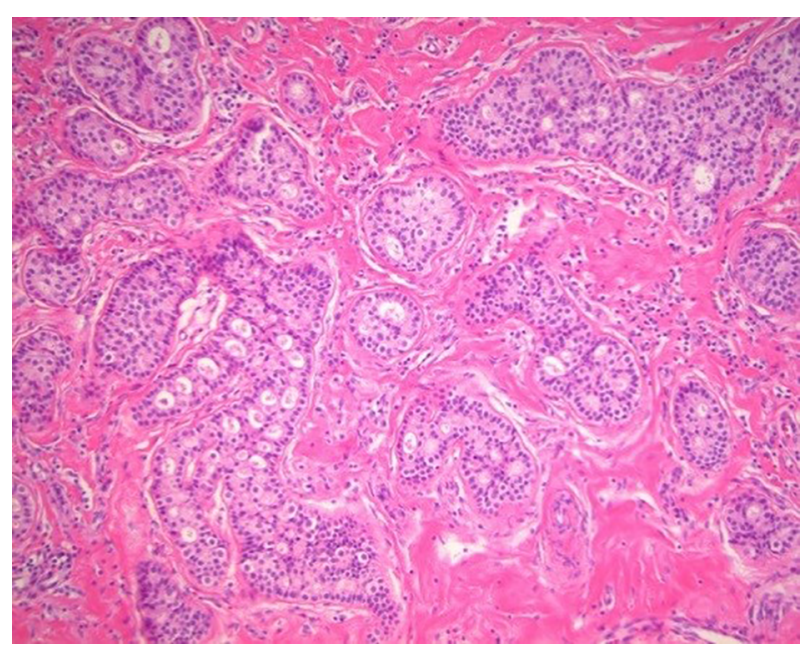

Figure 3. Low power microscopic findings (hematoxylin and eosin staining; magnification, $200 \times$ ) in the right breast mass. The tumor is arranged as invasive islands, in which well-defined spaces are formed by cribriform pattern. The tumor cells are small and show a low degree of nuclear pleomorphism with rare mitoses.

ular lesion on fine-needle aspiration that was finally diagnosed as benign after total thyroidectomy (Figure 4).

After 2 years, she underwent excision of the newly developed left chest wall mass on the skin layer. The pathologic diagnosis was an atypical fibro-histocytic lesion with no definite evidence of malignancy.

\section{Discussion}

Cowden syndrome is known as multiple hamartoma syndrome because a variety of benign, proliferative lesions are seen in various organs. The common conditions are benign proliferative lesions in the breast, thyroid, uterine, and skin nodules. This syndrome is associated with susceptibility to breast cancer, and is reported as the most common malignancy involving women with Cowden syndrome. In addition, bilateral breast cancers are reported in $25 \%$ of patients, so annual screening magnetic resonance imaging (MRI) is recommended (1, 2, 5, 8-13).

So far, there has been no reported cases of pure cribriform carcinoma in Cowden syndrome patients and the histologic subtype of breast cancer in has been rarely reported.

In a study conducted by Schrager et al. (2), among 14 patients of breast cancer, only one patient had ductal carcinoma in situ (DCIS) and 11 patients had both DCIS and invasive carcinoma. Among the 11 patients, only one patient had infiltrating tubular carcinoma and lobular carcinoma in situ (LCIS). The most common type of infiltrating carcinoma was ductal as well (86\%). All the patients hand mixed histologic subtypes of DCIS (assorted combination of cribriform, micropapillary, papillary, or solid).

There was a case report of synchronous bilateral breast carcinoma in Cowden syndrome. The tumor from the right breast consisted of DCIS of intermediate grade that was predominantly cribriform type. Different from the tumor in the right breast, the tumor in the left breast revealed DCIS of high-grade with various types, such as papillary, cribriform, solid, and comedo-type (14).

In our case, invasive ductal carcinoma was observed in the left breast showing a highly suspicious sonographic feature. On the other hand, a low suspicious mass with pathologic proven pure cribriform carcinoma was de- 

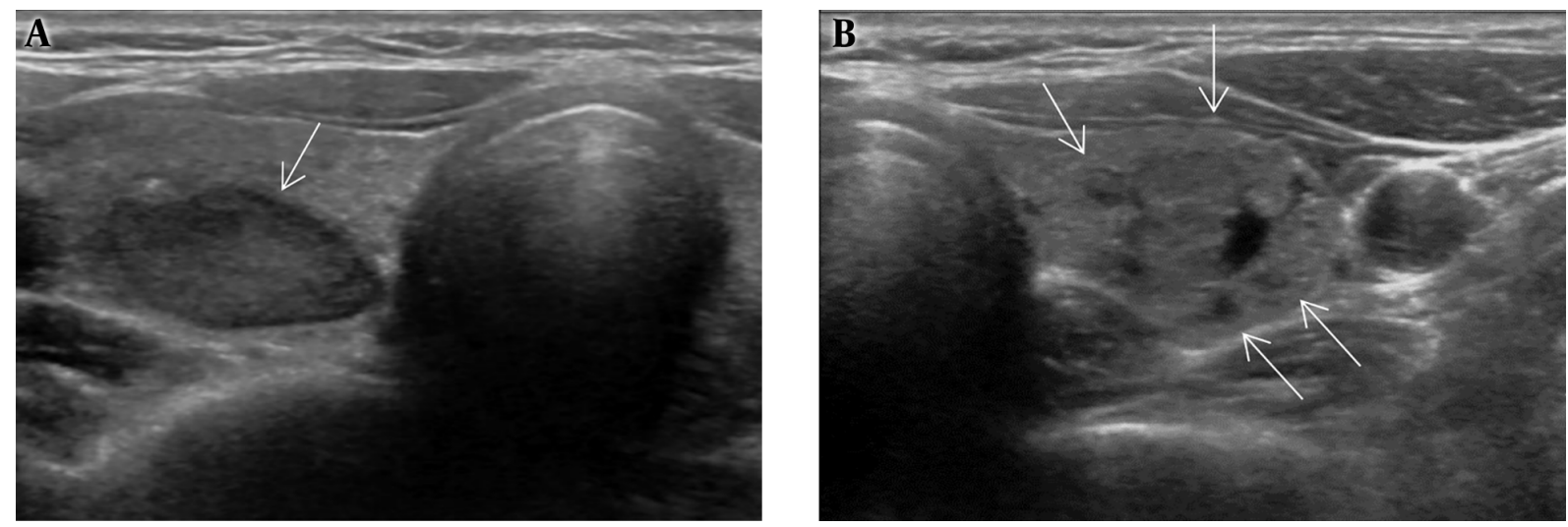

Figure 4. On thyroid ultrasonography, several ovoid solid and predominantly solid nodules are noted in both lobes of the thyroid glands (arrows).

tected in her right breast.

Page et al. (9) described infiltrating cribriform carcinoma of the breast, which is characterized by a predominant cribriform growth pattern of its invasive component, is a distinct histological type of invasive carcinoma. This is a rare condition of breast cancer, with an incidence of $0.3 \%$ to $3.5 \%$. Pure infiltrating cribriform carcinoma is defined as being almost entirely (>90\%) of an invasive cribriform pattern. The radiologic findings of infiltrating cribriform carcinoma are not well known since few studies have described the disease. The tumor was mammographically occult in $22 \%-50 \%$ of the patients (8-10). Therefore, sometimes the tumor is not definitely detected on mammography.

Especially the tumor occult sometimes on mammography (10). Sonographic assessment findings were classified as Breast Imaging Reporting and Data System(BI-RADS) category $4 \mathrm{~A}$ or $4 \mathrm{C}$. The carcinoma has a low frequency of axillary nodal metastases and a favorable prognosis $(8,11)$. In this case, mammography also did not reveal the mass, and the sonographic assessment findings were BI-RADS category $4 \mathrm{~A}$.

In conclusion, we have reviewed a case of Cowden syndrome in a woman diagnosed as invasive carcinoma of NST and contralateral cribriform carcinoma in the breast. There have been many reports of bilateral breast cancer with ductal cancer in patients with Cowden syndrome, and we report bilateral breast cancer with an exceptional pathology. Breast fibroadenomas, skin hemangiomas, gastric polyps, seborrheic hyperplasia, and benign thyroid lesions were also present in the patient.

\section{Footnotes}

Authors' Contributions: Go Eun Yang made substantial contributions to conception and design. Yoo Kyeong Seo was a major contributor in writing the manuscript. Literature review and data analysis was done by Taek Geun Ohk. All authors read and approved the final manuscript.

Conflict of Interests: None of the authors has any conflict of interest.

Funding/Support: There was no funding/support to declare.

Informed Consent: Written informed consent was obtained.

\section{References}

1. Pilarski R. Cowden syndrome: A critical review of the clinical literature. J Genet Couns. 2009;18(1):13-27. doi: 10.1007/s10897-008-9187-7. [PubMed: 18972196].

2. Schrager CA, Schneider D, Gruener AC, Tsou HC, Peacocke M. Clinical and pathological features of breast disease in Cowden's syndrome: an underrecognized syndrome with an increased risk of breast cancer. Hum Pathol. 1998;29(1):47-53. doi: 10.1016/s0046-8177(98)90389-6. [PubMed: 9445133].

3. Tan MH, Mester JL, Ngeow J, Rybicki LA, Orloff MS, Eng C. Lifetime cancer risks in individuals with germline PTEN mutations. Clin Cancer Res. 2012;18(2):400-7. doi: 10.1158/1078-0432.CCR-11-2283. [PubMed: 22252256]. [PubMed Central: PMC3261579].

4. Jung MH. Breast cancer in cowden syndrome: A case report. J Korean Soc Radiol. 2009;60(4):279. doi:10.3348/jksr.2009.60.4.279.

5. Seo M, Cho N, Ahn HS, Moon HG. Cowden syndrome presenting as breast cancer: Imaging and clinical features. Korean J Radiol. 2014;15(5):586-90. doi: 10.3348/kjr.2014.15.5.586. [PubMed: 25246819]. [PubMed Central: PMC4170159].

6. Sabate JM, Gomez A, Torrubia S, Blancas C, Sanchez G, Alonso MC, et al. Evaluation of breast involvement in relation to Cowden syndrome: A radiological and clinicopathological study of patients with PTEN germ-line mutations. Eur Radiol. 2006;16(3):702-6. doi: 10.1007/s00330-005-2877-8. [PubMed:16208511]. 
7. Koh J, Kim MJ. Introduction of a new staging system of breast cancer for radiologists: An emphasis on the prognostic stage. Korean J Radiol. 2019;20(1):69-82. doi: 10.3348/kjr.2018.0231. [PubMed: 30627023]. [PubMed Central: PMC6315072].

8. Cong Y, Qiao G, Zou H, Lin J, Wang X, Li X, et al. Invasive cribriform carcinoma of the breast: A report of nine cases and a review of the literature. Oncol Lett. 2015;9(4):1753-8. doi: 10.3892/ol.2015.2972. [PubMed: 25789036]. [PubMed Central: PMC4356388].

9. Page DL, Dixon JM, Anderson TJ, Lee D, Stewart HJ. Invasive cribriform carcinoma of the breast. Histopathology. 1983;7(4):525-36. doi: 10.1111/j.1365-2559.1983.tb02265.x. [PubMed: 6884999].

10. Stutz JA, Evans AJ, Pinder S, Ellis IO, Yeoman LJ, Wilson AR, et al. The radiological appearances of invasive cribriform carcinoma of the breast. Nottingham Breast Team. Clin Radiol. 1994;49(10):693-5. doi: 10.1016/s0009-9260(05)82662-5. [PubMed: 7955831].
11. Bubien V, Bonnet F, Brouste V, Hoppe S, Barouk-Simonet E, David A, et al. High cumulative risks of cancer in patients with PTEN hamartoma tumour syndrome. J Med Genet. 2013;50(4):255-63. doi: 10.1136/jmedgenet-2012-101339. [PubMed: 23335809].

12. Daly MB, Pilarski R, Berry M, Buys SS, Farmer M, Friedman S, et al. NCCN guidelines insights: Genetic/familial high-risk assessment: Breast and ovarian, version 2.2017. J Natl Compr Canc Netw. 2017;15(1):9-20. doi: 10.6004/jnccn.2017.0003. [PubMed: 28040716].

13. Hobert JA, Eng C. PTEN hamartoma tumor syndrome: An overview. Genet Med. 2009;11(10):687-94. doi: 10.1097/GIM.0b013e3181ac9aea. [PubMed: 19668082].

14. Kwon SY, Yeo SH, Ha JS, Kang SH. Synchronous bilateral breast carcinoma in a patient with cowden syndrome with Cowdon syndrome mutation: A case report. J Breast Dis. 2018;6(2):79-83. doi: 10.14449/jbd.2018.6.2.79. 